\title{
Startling speech: eliciting prepared speech using startling auditory stimulus
}

\author{
Chenhao Chiu ${ }^{1 *}$ and Bryan Gick ${ }^{1,2}$ \\ 1 Department of Linguistics, University of British Columbia, Vancouver, BC, Canada \\ 2 Haskins Laboratories, New Haven, CT, USA \\ *Correspondence: chenhao@alumni.ubc.ca
}

Edited by:

Eddy J. Davelaar, Birkbeck College, UK

Reviewed by:

Caroline A. Niziolek, University of California, San Francisco, USA

Keywords: speech planning, feedforward control, speech motor control, startling auditory stimulus, the StartReact effect

Speech research has recently seen a good deal of activity surrounding forward models (Tian and Poeppel, 2012; Pickering and Garrod, 2013; Scott, 2013), expanding on a long tradition of work in preprogramming of speech motor plans (e.g., Lashley, 1951; Keele, 1981; Klapp, 2003). Despite the volume of activity and interest in this area, few studies have offered insight into the detailed content of these forward plans. The content of such plans should presumably specify, at minimum, those aspects of speech that are essential in determining linguistic contrast, independent of the many aspects of a physical speech utterance that may be determined or altered through feedback mechanisms. Our previous work has attempted to uncover some of the detailed content of such forward plans using behavioral methods (Scott et al., 2013), while other studies have used neuroimaging methods (e.g., Heinks-Maldonado et al., 2006). Both approaches have given suggestive results, though not without concerns regarding interpretation (Niziolek et al., 2013).

A novel experimental methodology employing startling auditory stimuli (SAS, $>120 \mathrm{~dB}$ ) has been used to demonstrate the execution of prepared non-speech motor behaviors (e.g., head rotation and upper limb movements) with little or no interference from feedback regulation (Valls-Solé et al., 1999; Oude Nijhuis et al., 2007; Carlsen et al., 2012). Accelerated release of prepared movements (as short as $70 \mathrm{~ms}$ for EMG response onset) in response to SAS has been termed the StartReact effect (Valls-Solé et al., 1999,
2008). Because of their very short onset latency, SAS-induced actions may be fully executed before they are affected by sensory feedback, thus enabling study of the forward plan. It is our opinion that this experimental paradigm is ideally suited for investigating speech production and uncovering the detailed contents of forward speech plans.

Early analyses hypothesized that the rapid release of SAS-induced responses is the result of triggering subcortically stored information with faster neural transmissions (Carlsen et al., 2004; see also Castellote et al., 2012; Nonnekes et al., 2014). However, recent transcranial magnetic stimulation (TMS) studies show that the StartReact effect may not be limited to subcortically stored programs, but can also be observed in cortically dependent processes (Alibiglou and MacKinnon, 2012; Stevenson et al., 2014). These studies found that, when a cortical silent period was induced by applying TMS to motor cortex, the StartReact response was delayed in startle trials. If only subcortical processes were involved, TMS should not have affected the StartReact response. The delay in the StartReact response suggests that the pathways for a StartReact response would be mediated by, rather than bypassing, cortical areas.

Following these studies of finger movement, Stevenson et al. (2014) apply the startle paradigm to prepared spoken syllables, observing that voluntary lip movements were released at shorter latencies by a SAS, while the timing of kinematic displacement remained unaffected and formant profiles were performed as intended with no disruption. These results support the view that prepared syllables encode sufficient kinematic and acoustic information as part of the forward plan, and that this information may be subject to rapid release by a SAS. Extending this paradigm to pitch control in speech, Chiu and Gick (in press) show that a SAS induces an elevated pitch level in prepared syllables. Speakers show no evidence of an attempt to correct this elevated pitch to a baseline level even though auditory and somatosensory feedback is likely available before the end of the response. These findings raise questions as to the extent to which feedback information may affect SAS-induced responses, and suggest that uncorrected contents of forward speech plans may be observable even for longer (i.e., multisyllabic) responses using SAS.

The observed StartReact effect in syllable production also suggests that SAS-induced motor tasks, including upper limb, and speech movements, may involve similar neural pathways. It is noteworthy that the StartReact pathways involve similar pathways for speech production. As summarized in Carlsen et al. (2012), the StartReact response is mediated via an ascending thalamo-cortical pathway, generated by activation from reticular formation exerting on thalamus. Increased activation in thalamus provides inputs to primary motor cortex to initiate the cortically prepared movement via a descending corticospinal pathway. Similarly, speech production 
may also rely on thalamo-cortical circuits, and a descending corticospinal pathway. Specifically, receiving inputs from cerebellum, thalamus projects to primary motor cortex and Broca's area, and the commands are mediated via putamen and reticular formation and sent down to the phonatory motoneurones in the spine (Iwata et al., 1996; Jürgens, 2002; Guenther et al., 2006). Given that speech production involves a similar thalamo-cortical pathway to the one found in upper limb movements, upper limb movements, and speech movements may share the same StartReact pathways when elicited by a SAS. Shorter reaction times in StartReact responses are accounted for by increased neuron activation reaching faster above initiation threshold (see Carlsen et al., 2012 for details).

Similar to the calculation of the required time span for voluntary limbic movements, we can also conservatively calculate the time required for a speech response. First, Schroeder and Foxe (2002) report a response latency of $10 \sim 25 \mathrm{~ms}$ from the onset of auditory stimulus to the activation in the auditory cortex. Second, another $5 \sim 10 \mathrm{~ms}$ is required for the stimulus to be conducted between the lateral lemniscus and the thalamus for auditorilyevoked responses (Stockard et al., 1977). Third, transcortical and thalamus-primary motor cortex transmissions require $2 \sim$ $4 \mathrm{~ms}$ for conduction (Guenther et al., 2006; Carlsen et al., 2012). Last, the orofacial muscle EMG response to TMS on the face area of the motor cortex has a latency of about $11 \sim 12 \mathrm{~ms}$ (Meyer et al., 1994) and the motor time for the muscle movement is delayed by $30 \mathrm{~ms}$. Adding these values gives a minimum of $58 \sim 81 \mathrm{~ms} \mathrm{lag}$ time in response to a SAS. As reported in Stevenson et al. (2014), the onset of SASinduced responses is $75 \mathrm{~ms}$, suggesting that the shared neural pathway used for limb movements and speech movements does lead to a StartReact effect for speech movement.

Insofar as programming is necessary for speech production, we believe that the SAS methodology provides a new perspective that can help us to uncover the kinematic and linguistic contents of forward speech plans. Neural correlates and pathways for SAS-induced responses also support the view that SAS-induced speech responses may contain unaltered details of speech plans, allowing researchers a window into forward speech planning that bypasses afferent feedback information.

\section{ACKNOWLEDGMENT}

This work was supported by the National Institute of the Health Grant DC-02717 to Haskins Laboratories.

\section{REFERENCES}

Alibiglou, L., and MacKinnon, C. D. (2012). The early release of planned movement by acoustic startle can be delayed by transcranial magnetic stimulation over the motor cortex. J. Physiol. 590, 919-936. doi: 10.1113/jphysiol.2011. 219592

Carlsen, A. N., Chua, R., Inglis, T. J., Sanderson, D. J., and Franks, I. M. (2004). Can prepared responses be stored subcortically. Exp. Brain Res. 159, 301-309. doi: 10.1007/s00221-0041924-Z

Carlsen, A. N., Maslovat, D., and Franks, I. M. (2012). Preparation for voluntary movement in healthy and clinical populations: evidence from startle. Clin. Neurophysiol. 123, 21-33. doi: 10.1016/j.clinph.2011.04.028

Castellote, J. M., Queralt, A., and Valls-Solé, J. (2012). Preparedness for landing after a selfinitiated fall. J. Neurophysiol. 108, 2501-2508. doi: 10.1152/jn.01111.2011

Chiu, C., and Gick, B. (in press). Pitch planning in English and Taiwanese Mandarin: evidence from startle-elicited responses. J. Acoust. Soc. Am. Exp. Lett.

Guenther, F. H., Ghosh, S. S., and Tourville, J. A. (2006). Neural modeling and imaging of the cortical interactions underlying syllable production. Brain Lang. 96, 280-301. doi: 10.1016/j.bandl.2005.06.001

Heinks-Maldonado, T. H., Nagarajan, S. S., and Houde, J. F. (2006). Magnetoencephalographic evidence for a precise forward model in speech production. Neuroreport 17, 1375-1379. doi: 10.1097/01.wnr.0000233102.43526.e9

Iwata, K., Yagi, J., Tsuboi, Y., Koshikawa, N., Sumino, R., and Cools, A. R. (1996). Anatomical connections of the ventral but not the dorsal part of the striatum with the parvicellular reticulation formation: implications for the anatomical substrate of oral movements. Neurosci. Res. Commun. 18, 71-78. doi: 10.1002/(SICI)15206769(199603)18:2<71::AID-NRC142>3.0.CO;2-0

Jürgens, U. (2002). Neural pathways underlying vocal control. Neurosci. Biobehav. Rev. 26, 235-258. doi: 10.1016/S0149-7634(01)00068-9

Keele, S. W. (1981). "Behavioral analysis of movement," in Handbooks of Physiology: Sec. 1: The Nervous System: Vol. 2: Motor Control, ed V. Brooks (Baltimore, MD: Williams \& Wilkins), 1391-1414.

Klapp, S. T. (2003). Reaction time analysis of two types of motor preparation for speech articulation: actions as a sequence of chunks. J. Mot. Behav. 35, 135-150. doi: 10.1080/00222890309 602129
Lashley, K. S. (1951). "The problem of serial order in behavior," in Cerebral Mechanisms in Behavior, ed L. A. Jeffress (New York, NY: Wiley), 112-131.

Meyer, B. U., Werhahn, K., Rothwell, J. C., Roericht, S., and Fauth, C. (1994). Functional organisation of corticonuclear pathways to motoneurones of lower facial muscles in man. Exp. Brain Res. 101, 465-472. doi: 10.1007/BF002 27339

Niziolek, C. A., Nagarajan, S. S., and Houde, J. F. (2013). What does motor efference copy represent? Evidence from speech production.J. Neurosci. 33, 16110-16116. doi: 10.1523/JNEUROSCI.213713.2013

Nonnekes, J., Oude Nijhuis, L. B., de Niet, M., de Bot, S. T., Pasman, J. W., van de Warrenburg, B. P. C., et al. (2014). StartReact restores reaction time in HSP: evidence for subcortical release of a motor program. J. Neurosci. 34, 275-281. doi: 10.1523/JNEUROSCI.294813.2014

Oude Nijhuis, L. B., Janssen, L., Bloem, B. R., Gert van Dijk, J., Gielen, S. C., Borm, G. F., et al. (2007). Choice reaction times for human head rotations are shortened by startling acoustic stimuli, irrespective of stimulus direction. J. Physiol. 584, 97-109. doi: 10.1113/jphysiol.2007. 136291

Pickering, M. J., and Garrod, S. (2013). An integrated theory of language production and comprehension. Behav. Brain Sci. 36, 329-392. doi: 10.1017/S0140525X12001495

Schroeder, C. E., and Foxe, J. J. (2002). The timing and laminar profile of converging inputs to multisensory areas of the macaque neocortex. Cogn. Brain Res. 14, 187-198. doi: 10.1016/S09266410(02)00073-3

Scott, M. (2013). Corollary discharge provides the sensory content of inner speech. Psychol. Sci. 24, 1824-1830. doi: 10.1177/09567976134 78614

Scott, M., Yeung, H., Gick, B., and Werker, J. (2013). Inner speech captures the perception of external speech. J. Acoust. Soc. Am. 133, 286-292. doi: 10.1121/1.4794932

Stevenson, A. J., Chiu, C., Maslovat, D., Chua, R., Gick, B., Blouin, J.-S., et al. (2014). Cortical involvement in the StartReact effect. Neuroscience 269, 21-34. doi: 10.1016/j.neuroscie nce.2014.03.041

Stockard, J. J., Stockard, J. E., and Sharbrough, F. W. (1977). Detection and localization of occult lesions with brain-stem auditory responses. Mayo Clin. Proc. 52, 761-769.

Tian, X., and Poeppel, D. (2012). Mental imagery of speech: linking motor and perceptual systems through internal simulation and estimation. Front. Hum. Neurosci. 6:1-11. doi: 10.3389/fnhum.2012.00314

Valls-Solé, J., Kumru, H., and Kofler, M. (2008). Interaction between startle and voluntary reactions in humans. Exp. Brain Res. 187, 497-507. doi: 10.1007/s00221-008-1402-0

Valls-Solé, J., Rothwell, J. C., Goulart, F., Cossu, G., and Muñoz, E. (1999). Patterned ballistic movements triggered by a startle in healthy humans. J. Physiol. 516, 931-938. doi: 10.1111/j.14697793.1999.0931u.x 
Conflict of Interest Statement: The authors declare that the research was conducted in the absence of any commercial or financial relationships that could be construed as a potential conflict of interest.

Received: 26 June 2014; accepted: 08 September 2014; published online: 26 September 2014.
Citation: Chiu C and Gick B (2014) Startling speech: eliciting prepared speech using startling auditory stimulus. Front. Psychol. 5:1082. doi: 10.3389/fpsyg. 2014.01082

This article was submitted to Cognitive Science, a section of the journal Frontiers in Psychology.

Copyright (c) 2014 Chiu and Gick. This is an open-access article distributed under the terms of the
Creative Commons Attribution License (CC BY). The use, distribution or reproduction in other forums is permitted, provided the original author(s) or licensor are credited and that the original publication in this journal is cited, in accordance with accepted academic practice. No use, distribution or reproduction is permitted which does not comply with these terms. 